\title{
PROPERTIES AND SYNTHESIS OF HEAVY NUCLEI AND PROPERTIES OF NEUTRON STAR MATTER
}

\author{
J. ROBERT BUCHLER \\ Belfer Graduate School of Science, Yeshiva University, New York, N.Y., U.S.A.
}

\begin{abstract}
The nuclear Thomas-Fermi model which is based on nuclear matter calculations has been successfully applied to the study of the bulk properties of nuclei. It is ideally suited for extrapolation into the region of very neutron-rich and of superheavy nuclei. It is therefore a valuable approach for $r$-process calculations as well as for the study of neutron star matter at subnuclear densities.
\end{abstract}

Many physical situations require the knowledge of the properties of nuclei located far away from the region of stability. The standard semi-empirical mass formulae give a good fit to the known nuclei, but their extrapolation into the neutron-rich and superheavy region is subject to considerable uncertainty. This is due to the fact that only the coefficients of the four leading terms can be reliably fitted by a least squares procedure. In the neutron-rich regions, mass formula terms which are negligible in the vicinity of the beta stability valley, and therefore ill determined, can significantly influence estimates of nuclear properties. It is therefore of interest to have an alternate simple way to get a grasp on these nuclei without having to do, for instance, a full Brueckner-Hartree-Fock calculation.

The idea of reproducing the bulk properties of nuclei, i.e., their binding energies, radii and surface thicknesses, by means of a statistical or Thomas-Fermi approach, goes back to the earlier days of nuclear physics. The earlier approaches were semiphenomenological in the sense that a whole set of parameters had to be fitted to give agreement with experiment. The present idea is to make maximum use of the results of realistic nuclear matter calculations, i.e. the binding energy of a homogeneous mixture of neutrons and protons as a function of neutron excess and of density. This allows us to reduce the number of free parameters to just one.

The Thomas-Fermi approach is based on a theorem by Hohenberg and Kohn (1964) which states that for a many-body system the energy is a functional of the density alone and that the ground state density distribution is the one which minimizes this functional at constant number of particles. When the density is slowly varying, the functional may be expanded in terms of the gradients of the density. The exact form of the functional is of course unknown. According to Brueckner and collaborators (see, e.g., Brueckner et al. (1971) and references therein; Barkat et al. (1972)), we therefore approximate the energy of a nucleus by the statistical expression

$$
\begin{aligned}
E\left[\varrho_{\mathrm{n}}, \varrho_{\mathrm{p}}\right]= & \int(\mathrm{d} r)^{3}\left\{\varepsilon\left[\varrho_{\mathrm{n}}(r), \varrho_{\mathrm{p}}(r)\right]+\frac{e}{2} \varrho_{\mathrm{p}}(r) \Phi(r)+\right. \\
& \left.-\frac{3}{4}\left(\frac{3}{\pi}\right)^{1 / 3} e^{2}\left[\varrho_{\mathrm{p}}(r)\right]^{4 / 3}+\eta[\nabla \varrho(r)]^{2}\right\} .
\end{aligned}
$$


The first term $\varepsilon$ is a function of the densities and represents the energy per unit volume of homogeneous nuclear matter of neutron density $\varrho_{n}$ and proton density $\varrho_{p}$. We have used for $\varepsilon$ the nuclear matter results of Brueckner et al. (1968b), together with a recent neutron gas calculation by Buchler and Ingber (1971). The second and third terms are the direct and exchange Coulomb energies, where the Coulomb potential $\Phi(r)$ is of course a functional of $\varrho_{\mathrm{p}}$. Finally, the last term represents the gradient correction which takes into account the change in correlation energy due to the density inhomogeneity. Here $\varrho=\varrho_{\mathrm{n}}+\varrho_{\mathrm{p}}$ represents the total nucleon density. As shown by Bethe (1968), the quantity $\eta$ is simply related to the 'long range' part of the nuclear force and should be only weakly dependent on density. In view of the uncertainty involved in calculating $\eta$, we have chosen to treat it as a constant determined by a fit to the nuclear binding energies over the range of known nuclei. The constant $\eta$ is the only phenomenological parameter of the Thomas-Fermi model and its fitted value is in good agreement with Bethe's theoretical estimate.

The problem now consists in minimizing the quantity

$$
E\left[\varrho_{\mathrm{n}}, \varrho_{\mathrm{p}}\right]-\lambda_{\mathrm{n}} \int \varrho_{\mathrm{n}}(r)(\mathrm{d} r)^{3}-\lambda_{\mathrm{p}} \int \varrho_{\mathrm{p}}(r)(\mathrm{d} r)^{3},
$$

where the Lagrange parameters $\lambda_{n}$ and $\lambda_{p}$ are the neutron and proton chemical potentials, respectively, and are chosen so that

$$
\int \varrho_{\mathrm{n}}(r)(\mathrm{d} r)^{3}=N, \text { and, } \int \varrho_{\mathrm{p}}(r)(\mathrm{d} r)^{3}=Z .
$$

This minimization leads to a system of non-linear equations of the form

$$
\begin{aligned}
2 \eta \nabla^{2} \varrho & =\frac{\partial \varepsilon}{\partial \varrho_{\mathrm{n}}}+\frac{\partial \varepsilon}{\partial \varrho_{\mathrm{p}}}+e \Phi-\left(\frac{3}{\pi}\right)^{1 / 3} e^{2} \varrho_{\mathrm{p}}^{1 / 3}+\lambda_{\mathrm{n}}+\lambda_{\mathrm{p}}, \\
0 & =\frac{\partial \varepsilon}{\partial \varrho_{\mathrm{n}}}-\frac{\partial \varepsilon}{\partial \varrho_{\mathrm{p}}}-e \Phi+\left(\frac{3}{\pi}\right)^{1 / 3} e^{2} \varrho_{\mathrm{p}}^{1 / 3}+\lambda_{\mathrm{n}}-\lambda_{\mathrm{p}} \\
\nabla^{2} \Phi & =-4 \pi e \varrho_{\mathrm{p}}
\end{aligned}
$$

with the condition that the densities $\varrho(r)$ have a continuous derivative everywhere. As a boundary condition, we require that $\mathrm{d} \varrho /\left.\mathrm{d} r\right|_{r=R}=0$, where $R$ represents the point where the density vanishes.

The density profiles for a series of nuclei are shown in Figure 1 . The ${ }^{16} \mathrm{O}$ nucleus is seen to consist mostly of surface; its central density never reaches the saturation density of nuclear matter $\left(0.2 \mathrm{fm}^{-3}\right)$. The heavier nuclei exhibit a dip at the center and a bulge in the outer regions due to the Coulomb repulsion between the protons. The protons also provoke a somewhat smaller bulge in the neutron density because of the symmetry energy.

The vanishing of the density rather than an exponential drop is typical of the Thomas-Fermi model and is a drawback as far as the study of the tails of the distribution are concerned, and in particular the study of the neutron halo. The proton density 
with our functional (1) vanishes with non-zero slope (for proton-rich nuclei it is the neutron density which vanishes with non-zero slope). This could be remedied by including in the energy functional a density difference gradient term of the form $\theta\left\{\nabla\left[\varrho_{\mathrm{n}}(r)-\varrho_{\mathrm{p}}(r)\right]\right\}^{2}$. Because of numerical difficulties, such a term has been treated as a correction to the energy rather than having been included in the minimization of the functional. Higher order terms in the gradient expansion have been omitted. The a postiori justification is that their contribution to the energy is negligible for the density profiles which we have obtained.

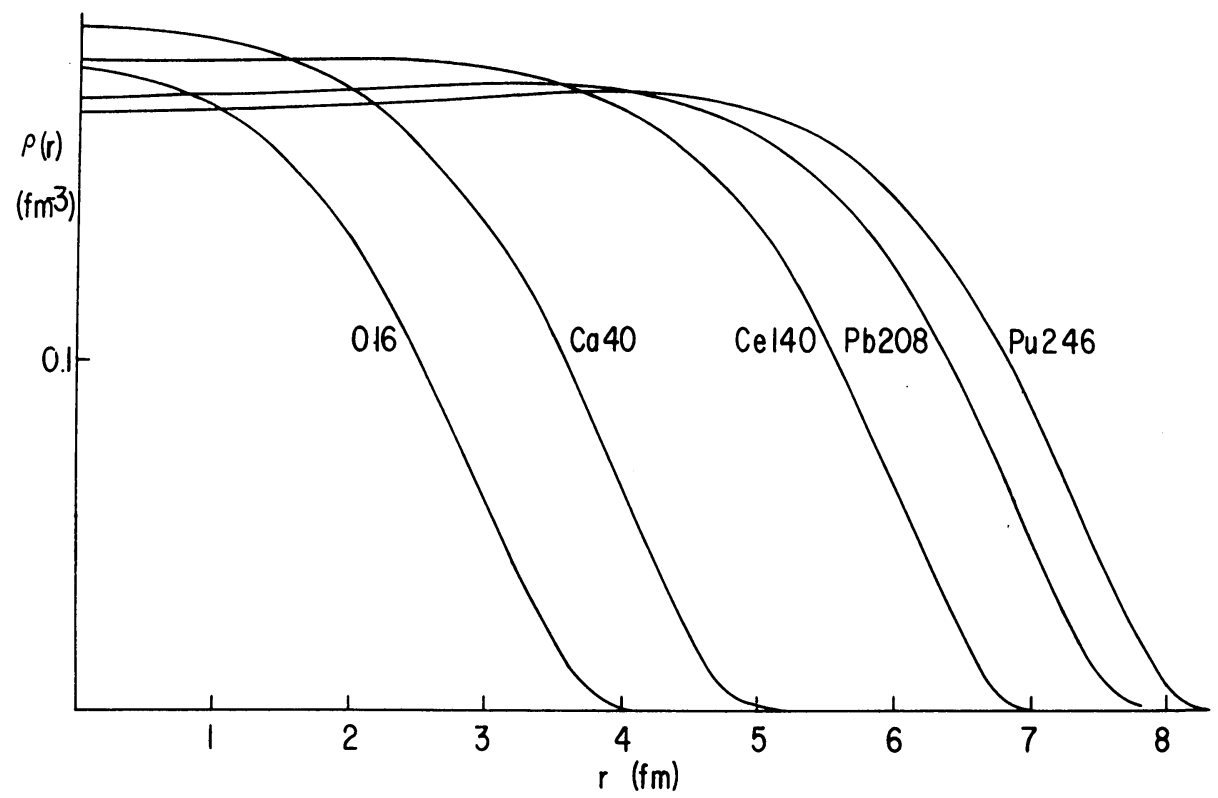

Fig. 1. Density profiles for various nuclei.

The computed binding energies per particle for the known spherical nuclei are in good agreement, to within $0.1 \mathrm{MeV}$ (except for ${ }^{16} \mathrm{O}$ for which the discrepancy is $\sim 0.5 \mathrm{MeV}$ ) with the liquid drop mass formula of Myers and Swiatecki. (Since our Thomas-Fermi model is a semi-classical approach which cannot reproduce shell effects, our results should not be compared to the actual binding energies of nuclei but to their 'liquid drop' part.) The proton radii turn out to be somewhat (a few percent) smaller and the surface diffuseness somewhat (5-10\%) larger than the electron scattering experiments seem to indicate (Brueckner et al., 1968a). The overall agreement, however, is remarkable considering that we have only one parameter in our theory.

Binding energy contours are shown in Figure 4 of Brueckner et al. (1971). In the region of known isotopes $(Z \leqslant 90, N \leqslant 160)$ including the transuraniums, the agreement is quite good. Deviations between the liquid drop model predictions and the Thomas-Fermi results are quite significant for neutron-rich and superheavy nuclei. 
Deformed nuclei have also been studied by Brueckner et al. (1970) with the restriction to ellipsoidal shapes. The density profiles exhibit a uniform surface diffuseness, as expected. For small deformations the binding energies are quite close to those of the liquid droplet models.

With this success of the Thomas-Fermi model at hand, we feel confident in extrapolating to the region of unknown nuclei. I will go on to describe two different applications: first, the possibility of producing superheavy nuclei in an $r$-process; and second, the nature of clusters of nucleons in neutron star matter.

In order to investigate the possible synthesis of superheavy elements during astrophysical processes, Brueckner et al. (1972) have recently incorporated the nuclear Thomas-Fermi binding energies in an $r$-process code by Seeger and Schramm. Their preliminary results show that, with the introduction of these Thomas-Fermi masses, significant changes occur in the results of $r$-process calculations. Most important, all indicate a strongly decreased probability for superheavy abundances relative to calculations based on the 'Myers-Swiatecki' mass formula. In the medium-heavy to superheavy element region, the $r$-process path with the energy density input is about 3 nucleon numbers closer to beta stability than the path corresponding to conventional mass formulas. This causes a pronounced shift in the isotopes of maximum abundance (the 'center line' in the $r$-process path). With the exception of the $N=184$ closed shell, the isotope of maximum abundance for a given $Z$ is about 5-10 neutrons poorer than those calculated with the Myers-Swiatecki mass formula. When the $r$-process is terminated due to fission, there are fewer neutron-rich nuclei available to beta decay into the superheavy region, so that the overall yield of superheavies is decreased.

At present, there is a controversy about the realistic value of the parameter $\kappa$, which is the ratio of the surface symmetry coefficient to the regular surface energy coefficient. Calculations were therefore performed for both the value of Myers and Swiatecki, $\kappa=1.79$, and a larger value of $\kappa=2.84$. The differences in the results for the two values of $\kappa$ are as follows: whereas with $\kappa=1.79$ one could hope for superheavy element production in the $r$-process, one cannot imagine this with $\kappa=2.84$. For this large value of $\kappa$ the barriers are just too small to allow any high $Z$, neutron-rich nuclei to survive. Even for the small $\kappa$ value, the abundances were smaller than those computed by Schramm and Fowler (1971). At the same temperature and density, the $r$-process cycle time is more than double, and the abundances of most of the isotopes of the element 112 are reduced by more than four orders of magnitude. However, it is possible to almost equal the superheavy abundances of Schramm and Fowler by increasing the neutron flux four orders of magnitude and decreasing the temperature by $10 \%$.

The other application of the Thomas-Fermi model which I will describe is the study of neutron star matter. By neutron star matter we mean cold, neutral, and catalyzed matter; cold, because the typical thermal energy of neutron stars is several orders smaller than the chemical potentials of the constituent particles; catalyzed, because we restrict ourselves to densities for which the relaxation time to complete equilibrium with respect to strong, electromagnetic and weak interactions is small. In other words, 
the question we ask is the following: at a given average baryon density, what particles are contained in the system and how do they spatially arrange themselves in their ground state configuration. It is well known that at low densities $\left(\varrho \lesssim 10^{6} \mathrm{~g} \mathrm{~cm}^{-3}\right)$ neutron star matter consists of ${ }^{56} \mathrm{Fe}$ (or ${ }^{62} \mathrm{Ni}$ ) nuclei arranged in a crystalline structure surrounded by a sea of electrons. At a density of about $10^{7} \mathrm{~g} \mathrm{~cm}^{-3}$, the chemical potential of the electrons reaches $1 \mathrm{MeV}$ and it becomes energetically favorable for protons to beta-capture; there results a gradual neutron enrichment of the nuclei as the density of matter increases. Since too great an enrichment would excessively raise the symmetry energy, there is at first a concomitant increase in the proton number of the nuclei. The chemical potential of the neutrons becomes positive at a density of about $3.7 \times 10^{11} \mathrm{~g} \mathrm{~cm}^{-3}$, so that some of the neutrons become unbound and form an embedding sea. At a density of about $10^{14} \mathrm{~g} \mathrm{~cm}^{-3}$ the clusters disappear and the system becomes a homogeneous mixture of neutrons, protons, and electrons.

In previous studies of neutron star matter in the clustered phase (cf. review articles by Cameron (1970), and Canuto (1973), it has been assumed that the clusters resemble ordinary nuclei sufficiently well that they can be described by a semi-empirical mass formula, or more recently by a compressible liquid droplet model with a variable surface coefficient to take into account the reduction of surface energy due to the surrounding neutron gas (Baym et al., 1971). Such an approach, however, suffers from the disadvantage that the results one obtains are very sensitive to the specific functional dependence of the mass formula on $A$ and $Z$. It also implies a dichotomy between a sharply defined nucleus and a surrounding gas. The Thomas-Fermi model, on the other hand, allows a consistent treatment of the nuclear surface by permitting the density profiles of the nuclei to change and to adjust smoothly to the embedding neutron fluid.

We will first concern ourselves with the clustered regime. Clustering occurs because the protons want to make maximum use of nuclear symmetry energy. In the spirit of the Wigner-Seitz model, Buchler and Barkat $(1971 \mathrm{a}, \mathrm{b})$ therefore assume that the system is composed of identical non-interacting spherical cells which are centered on the bulges of the proton distribution and which are electrically neutral. The energy per cell is then expressed in terms of the same integral Thomas-Fermi expression as previously described [Equation (1)], but supplemented by the electrons' energy. The radius of the cells is determined by the requirement of charge neutrality, which is the new constraint, instead of (3). The Coulomb lattice energy is thus automatically taken into account to good accuracy. The minimization of the energy functional has now to be performed with respect to the neutron, proton, and electron densities, and proceeds as previously. Some of the resulting densities as a function of distance from the center of the cell are shown in Figure 2. The numbers near the center denote the chemical potential of the neutrons (in $\mathrm{MeV}$ ), which is a monotonically increasing function of the overall matter density. The numbers in parentheses denote the radius of the cell (in $\mathrm{fm}$ ) which shrinks with increasing density. Note a decrease of the central densities and an increase in the surface diffuseness of the clusters with increasing outside neutron density, indicating a gradual dissolution of the clusters. In Figure 3 we show 


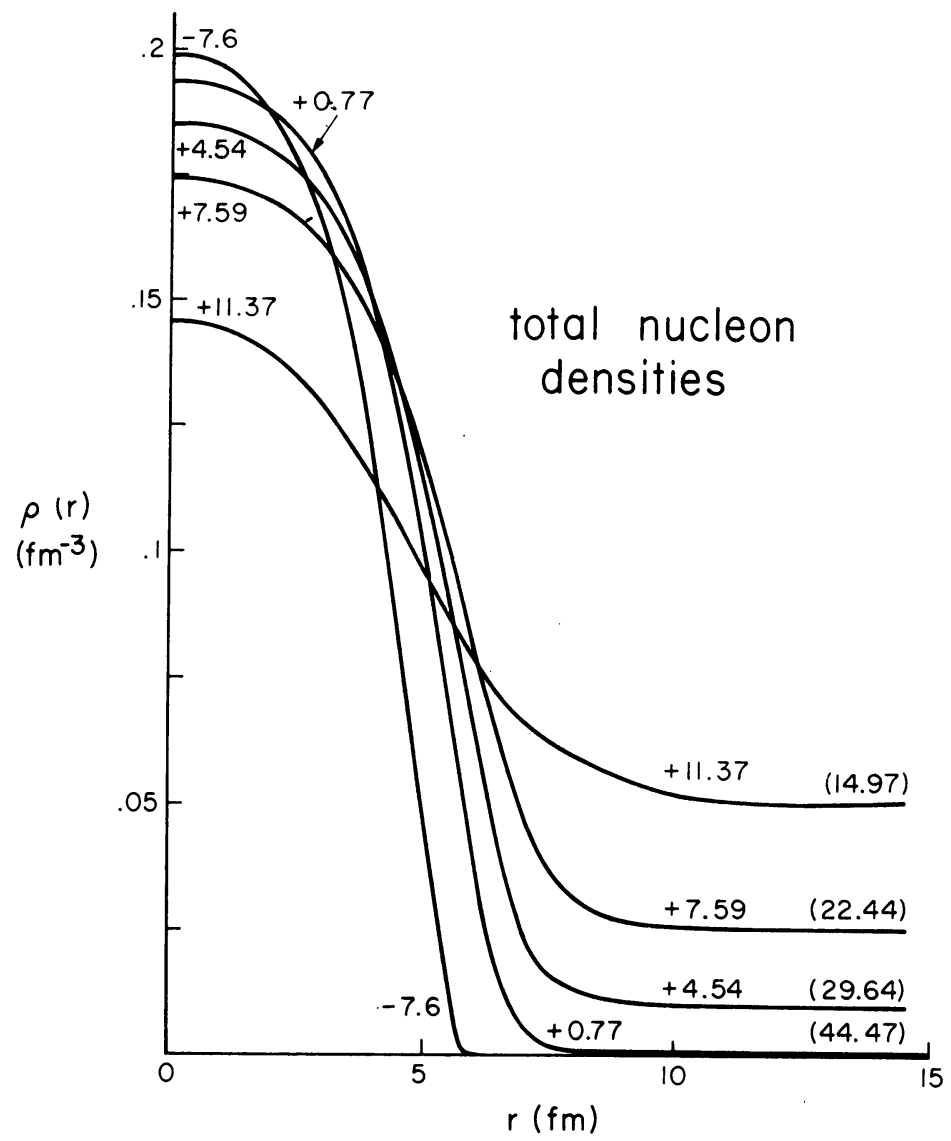

Fig. 2a. Total nucleon density distributions $Q(r)$ as a function of radial distance, labelled according to the corresponding neutron chemical potential $\lambda_{\mathrm{n}}$. Shown in parentheses are the cell radii in $\mathrm{fm}$.

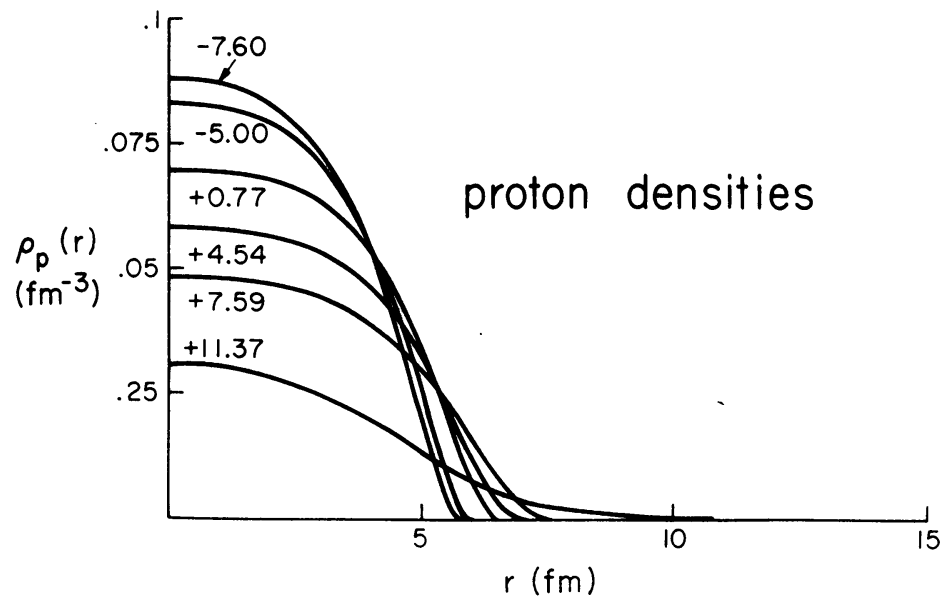

Fig. 2b. Proton density distribution $\varrho_{\mathrm{p}}(r)$. 
the dependence of $Z, A$, and of the density $\varrho$ on the chemical potential $\lambda_{\mathrm{n}}$ of the neutrons. The proton number $Z$ of the clusters first increases from $Z \simeq 29$ to a maximum of $Z \simeq 35$ and then decreases until the clusters gradually evanesce. Our calculations have not been carried beyond our last computed point because our approximation of spherical symmetry of the cells breaks down. Concurrently with us, Bethe et al. (1970) and later Baym et al. (1971) have been finding much larger nuclei ( $Z$ increasing to 151). This controversy has very recently been resolved; Ravenhall et al. (1972), using a one-dimensional (planar) model for the nuclear surface together with the sim-

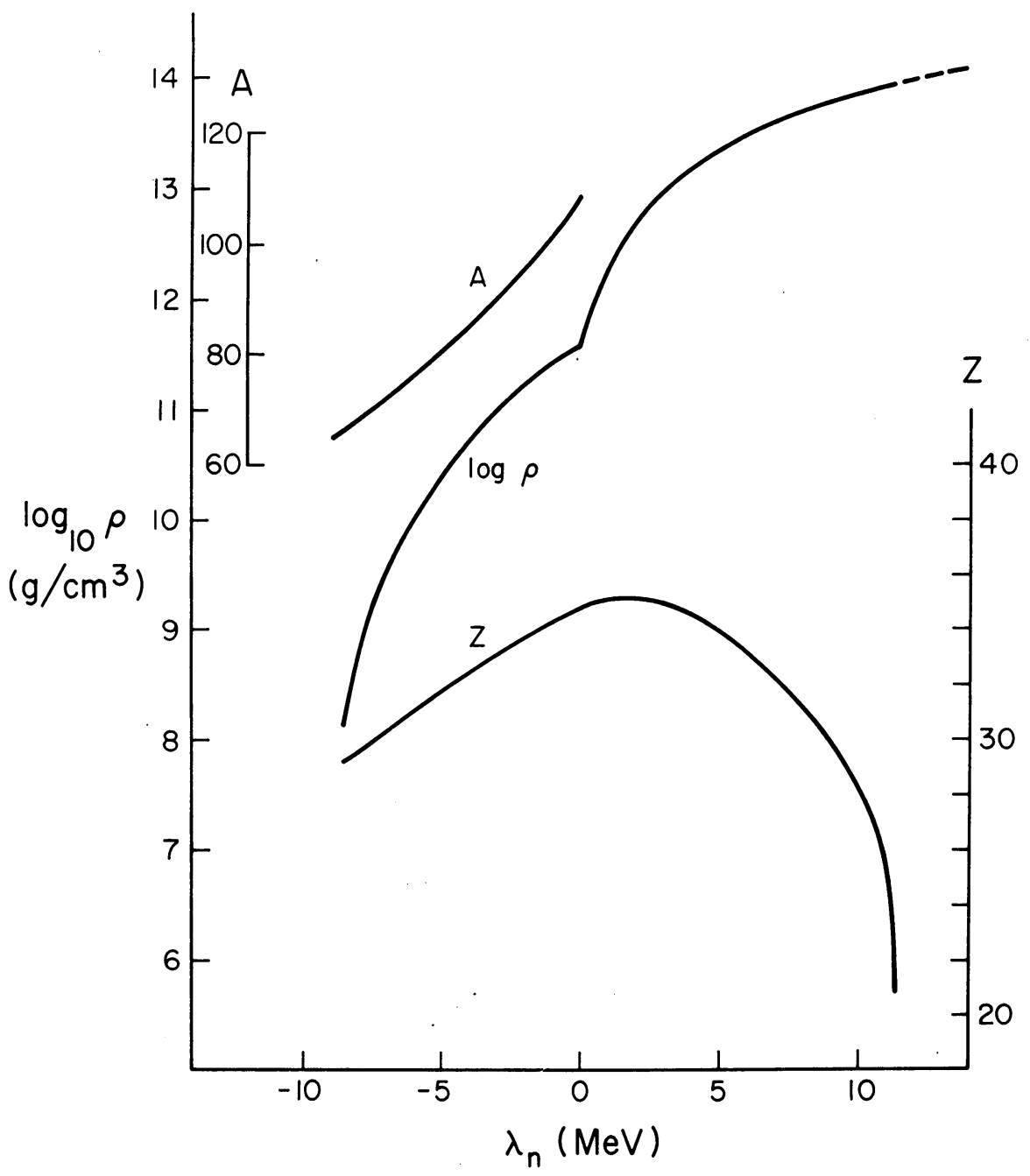

Fig. 3. Proton number $Z$, atomic number $A$ of the clusters, and average $\log _{10}$ (mass density in $\mathrm{g} \mathrm{cm}^{-3}$ ) as a function of the neutron chemical potential $\lambda_{\mathrm{n}}$. The dashed line corresponds to the homogeneous system, showing that the transition is smooth, which contrasts the 'neutron drip' phase transition $\left(\lambda_{\mathrm{n}}=0\right)$ at $3.7 \times 10^{11} \mathrm{~g} \mathrm{~cm}^{-3}$. 
ple Skyrme nucleon-nucleon interaction, also find very small nuclei $(Z \leqslant 38)$. It is gratifying that a Hartree-Fock calculation (cf. paper by Negele in this volume), which is much more involved and time-consuming, when averaged over shell effects, gives results in good qualitative agreement with our Thomas-Fermi calculation. The small quantitative discrepancy can probably be accounted for by the use of a different nucleonnucleon force and different nuclear matter results on which both approaches rely.

At higher densities, the clusters have dissolved and we have a homogeneous system of neutrons, protons, and electrons. The various physical quantities, like the energy per particle, the chemical potential of the protons, and the average neutron and proton densities join smoothly with the corresponding quantities of the homogeneous phase, as the calculations by Buchler and Ingber (1971) indicate. Although the exact nature of the phase transition is still uncertain, numerically it seems to be very smooth in contrast to the 'neutron drip' transition which occurred at $3.7 \times 10^{11} \mathrm{~g} \mathrm{~cm}^{-3}\left(\lambda_{\mathrm{n}}=0\right)$. In addition to the neutrons, protons, and electrons, muons also appear in the system at a density of $2.3 \times 10^{14} \mathrm{~g} \mathrm{~cm}^{-3}$, i.e., slightly before nuclear matter density $\left(3.4 \times 10^{14} \mathrm{~g}\right.$ $\mathrm{cm}^{-3}$ ). The percentage of protons increased from about $2.25 \%$ at $10^{14} \mathrm{~g} \mathrm{~cm}^{-3}$ to $5.5 \%$ at $4 \times 10^{14} \mathrm{~g} \mathrm{~cm}^{-3}$. It should be pointed out that these numbers are very sensitive to the symmetry energy of the nuclear matter calculations and the nucleon-nucleon potential used in its computation.

Shell effects have necessarily been ignored in our statistical approach, but it is likely that they may produce shifts and jumps in $Z$. Since a direct confrontation with observation is not possible, the final test for the Thomas-Fermi calculation will be a good quantum mechanical treatment. However, even a reliable Brueckner-HartreeFock calculation, because of its far greater complexity, is barely feasible at present.

In summary, the Thomas-Fermi model is capable of reproducing the bulk properties of a wide range of nuclei. It has the advantage of being based on realistic nuclear matter calculations and, therefore, can easily be kept $a$ jour with improvements in the nucleon-nucleon potential. We feel that the reliability of its predictions in $r$-process calculations and in the properties of neutron star matter by far exceeds that of conventional semi-phenomenological liquid-drop formulas.

This concludes our brief review of the nuclear Thomas-Fermi model and of some of its applications of astrophysical interest.

We wish to thank the authors of Brueckner et al. (1972) for letting us report their results prior to publication. The support of NSF, NASA and the Luxembourg Ministère des Affaires Culturelles is gratefully acknowledged.

\section{References}

Barkat, Z., Buchler, J. R., and Ingber, L.: 1972, Astrophys. J. 176, 723.

Baym, G., Bethe, H. A., and Pethick, C. J.: 1971, Nucl. Phys. A175, 225.

Bethe, H. A.: 1968, Phys. Rev. 167, 879.

Bethe, H. A., Börner, G., and Sato, K.: 1970, Astron. Astrophys. 7, 279.

Brueckner, K. A., Buchler, J. R., Clark, R. C., and Lombard, R. J.: 1968a, Phys. Rev. $181,1543$. 
Brueckner, K. A., Coon, S. A., and Dabrowski, J.: 1968b, Phys. Rev. 168, 1184.

Brueckner, K. A., Clark, R. C., Lin, W. F., and Lombard, R. J.: 1970, Phys. Rev. C1, 249.

Brueckner, K. A., Chirico, J. H., and Meldner, H. W.: 1971, Phys. Rev. C4, 732.

Brueckner, K. A., Chirico, J. H., Jorna, S., Meldner, H. W., Schramm, D. N., and Seeger, P. A.: 1972 (in preparation).

Buchler, J. R. and Barkat, Z.: 1971a, Astrophys. Letters 7, 169.

Buchler, J. R. and Barkat, Z.: 1971b, Phys. Rev. Letters 27, 48.

Buchler, J. R. and Ingber, L.: 1971, Nucl. Phys. A170, 1.

Cameron, A. G. W.: 1970, Ann. Rev. Astron. Astrophys. 8, 179.

Canuto, V.: 1973, Ann. Rev. Astron. Astrophys. (in press)

Hohenberg, P. C., and Kohn, W.: 1964, Phys. Rev. 136, B864.

Ravenhall, D. G., Bennett, C. D., and Pethick, C. J.: 1972, Phys. Rev. Letters 28, 978.

Schramm, D. N. and Fowler, W. A.: 1971, Nature 231, 103.

\section{DISCUSSION}

Bethe: The decision between our theories, in my opinion, has been made by the work reported by Dr Negele in the first paper of this conference. At low densities, of order $10^{12}-10^{13}, Z$ is 40 to 50 , closer to the original value of Baym, Bethe and Pethick than to yours. At high density, near $10^{14}$, the value of $Z$ decreases, as you have predicted, but not as strongly.

Buchler: I would like to refer back to the graph in Negele's talk which compared the $Z$ values for the different approaches. Negele's results, to me at least, seem to be in good qualitative agreement with ours. The discrepancy, I believe, could arise from the use of different nuclear matter calculations by him and by us; in particular, the arbitrary multiplication factor used by Negele to bring the nuclear binding energy in agreement with the currently accepted experimental value.

Bethe: The work of Negele and Vautherin, published in the Physical Review (C5), has shown that it is not sufficient to describe nuclear matter by $Q$ and $\nabla^{2} \varrho$ as you (and earlier Thomas-Fermi theories) have done, but that one has to add a third quantity, the kinetic-energy density

$$
t=\sum_{k}\left(\nabla \phi_{k}\right)^{2}
$$

which is independent of the first two. They have shown how this follows from a careful consideration of the mixed density $\varrho\left(\mathbf{r}_{1}, \mathbf{r}_{2}\right)$ which is very important for nuclear matter energy.

Buchler: As far as the second question is concerned, the Thomas-Fermi inhomogeneity correction to the kinetic energy, which arises purely from antisymmetrization of the wavefunction, is the wellknown Weizsäcker correction

$$
\frac{\hbar^{2}}{72 \mathrm{M}}\left[\frac{\left(\nabla \varrho_{\mathrm{n}}\right)^{2}}{\varrho_{\mathrm{n}}}+\frac{\left(\nabla Q_{\mathrm{p}}\right)^{2}}{\varrho_{\mathrm{p}}}\right] \equiv \frac{\hbar^{2}}{72 M} \frac{(\nabla \varrho)^{2}}{\varrho} .
$$

It plays essentially the same role as the term you mentioned. Its numerical effect has been found to be small and we feel that a 'renormalization' of our parameter $\eta$ should adequately take care of it. We realize that the Thomas-Fermi model is a very simple model and has its limitations, but its very simplicity and success make it very attractive. 